\title{
EUS Staging of Luminal Cancers in the Upper GI Tract
}

\author{
Juan Carlos Bucobo and Jonathan M. Buscaglia \\ Stony Brook University Medical Center \\ State University of New York at Stony Brook \\ Stony Brook, New York \\ USA
}

\section{Introduction}

Endoscopic ultrasound (EUS) has revolutionized the field of gastrointestinal endoscopy, and it plays a pivotal role in the staging of tumors of the upper gastrointestinal (GI) tract. EUS provides high-resolution imaging of both intraluminal and extraluminal structures, allowing for the detection and staging of even the smallest tumors in a minimally invasive manner. The accuracy of EUS in assessing depth of invasion of luminal tumors (T stage) is greater than other imaging modalities, including multi-detector CT and MRI. The addition of fine needle aspiration (FNA) allows for assessment of nodal involvement ( $\mathrm{N}$ stage), and although the quality of images obtained by CT and MRI continue to rapidly advance, EUS remains essential for obtaining tissue samples and for the definitive diagnosis of lesions within or adjacent to the upper GI tract.

This chapter aims to serve as an evidence-based and comprehensive review used to guide endosonographers involved in the care of patients with upper GI tract malignancies. We will outline the goals, challenges, and pitfalls encountered during the EUS evaluation for cancer staging. Furthermore, we will discuss the endoscopic technique best utilized for assessing cancers of the esophagus, stomach, ampulla and duodenum.

\section{Basic EUS principles for staging upper GI tumors}

The expertise of the endosonographer is considered one of the most important aspects in the staging of upper GI tract malignancy. A complete understanding of the normal anatomy and common congenital anomalies of the organ studied is a prerequisite for an accurate examination. Experience in identifying local and regional lymph nodes, and precisely assessing the level of tumor invasion, is critical for directing patients into the appropriate treatment algorithm; as often the choice of surgical procedure relies upon this information. There are several classification systems currently in use for defining GI cancers, predicting prognosis and determining treatment. Most commonly used is the tumor, node, metastasis (TNM) staging system as described by the American Joint Committee on Cancer (AJCC) [1]. The basic technique for performing endoscopic ultrasound for staging is similar for tumors located in the esophagus, stomach, and duodenum. We usually begin with standard upper endoscopy to visually assess the lesion, to determine whether the larger echoendoscope will 
be able to traverse the lesion, and to dilate any luminal malignant strictures if necessary. The location of the tumor being studied will often direct the choice of radial or linear echoendoscope, or whether a high-frequency ultrasound (HFUS) probe is needed. HFUS probes are ultrasound transducers located at the tip of a small-caliber ( 2 to $2.9 \mathrm{~mm}$ ) catheter that can pass through the working channel of a standard endoscope. They are available in frequencies between 12-30 MHz and yield high-resolution images of the gastrointestinal wall layers. They are typically used in the evaluation and $\mathrm{T}$ staging of small or superficial masses. Several studies have shown equivalency to conventional EUS for T staging although conventional EUS is superior for nodal staging[2].

In order to assess depth of invasion (T stage) for luminal tumors, it is necessary to identify 5 layers of wall structure that correspond to the histological layers (Image 1). HFUS allows for the visualization of up to nine layers. Using an echoendoscope at a frequency between 7.5 and $10 \mathrm{MHz}$, the 5 layers appear as alternating hyperechoic and hypoechoic bands as follows [3, 4]:

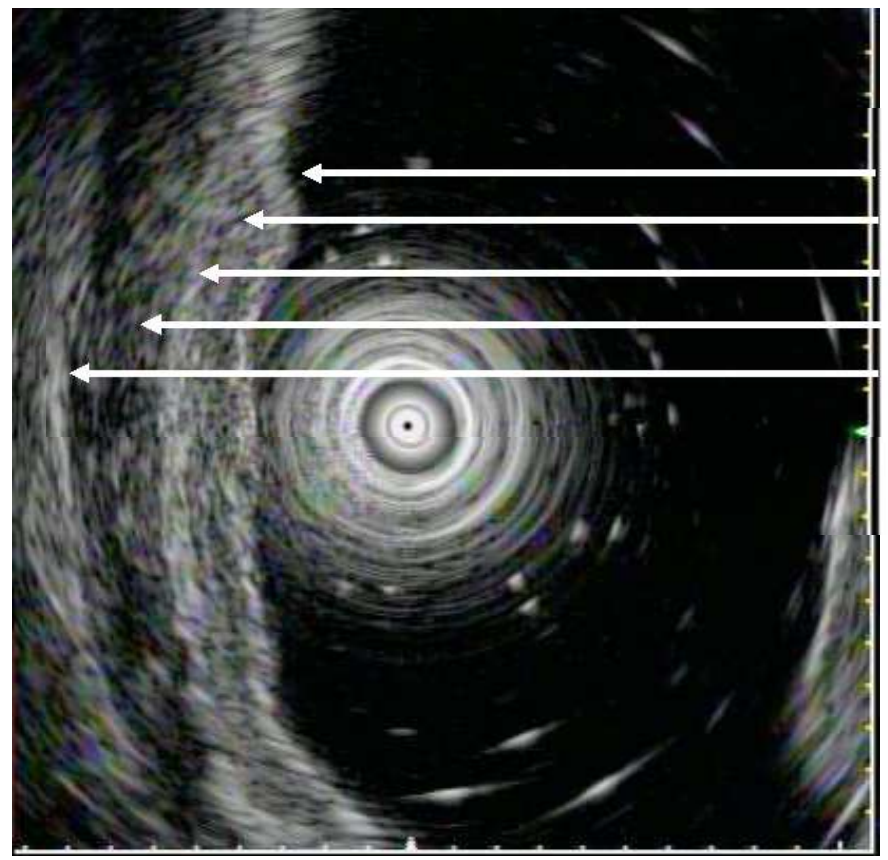

$1^{\text {st }}$ hyperechoic layer $2^{\text {nd }}$ hypoechoic layer $3^{\text {rd }}$ hyperechoic layer $4^{\text {th }}$ hypoechoic layer $5^{\text {th }}$ hyperechoic layer

$1^{\text {st }}$ hyperechoic layer: surface mucosa

$2^{\text {nd }}$ hypoechoic layer: deep mucosa

$3^{\text {rd }}$ hyperechoic layer: submucosa

$4^{\text {th }}$ hypoechoic layer: muscularis propria

$5^{\text {th }}$ hyperechoic layer: serosa (or adventitia in the esophagus)

Image 1. High-frequency probe demonstrating the layers of the gastric wall.

The EUS characteristics of lymph nodes for prediction of lymph node metastases ( $\mathrm{N}$ stage) were originally described in patients with esophageal cancer. Features suggestive of metastatic lymph nodes are size greater than $1 \mathrm{~cm}$, sharp borders, rounded shape, and 
homogenous hypoechoic echo pattern. When all four of these predictive features were found in a single lymph node, lymph node metastases were found in $100 \%$ of cases [5]. EUS fine-needle aspiration (FNA) adds to the evaluation for malignant lymph nodes by safely and accurately providing tissue samples which can influence patient management [6].

\section{Staging of esophageal tumors}

Esophageal tumors commonly present with symptoms of dysphagia and are often initially diagnosed by upper endoscopy. EUS provides superior loco-regional staging compared to other imaging modalities, and combined with FNA improves decision making regarding surgery and neo-adjuvant therapy [7, 8]. The role of EUS in early esophageal neoplasia is more controversial as endoscopic mucosal resection (EMR) may play an important role.

CT is usually performed early in the staging of esophageal cancers to evaluate for evidence of metastatic disease. EUS is indicated for staging of esophageal cancers in the absence of distant metastases on initial CT scan or MRI. Determining a T stage is accomplished by EUS and the first step for the endosonographer is repeating the standard upper endoscopy. This will allow the endosonographer to document the location of the tumor, measure the extent of stricture and assess whether the echoendoscope will easily pass the tumor if a stricture exists. Inability to pass the echoendoscope beyond a stricture is generally associated with a poorer prognosis as it may suggest advanced disease, but an effort should be made to traverse strictures as a more accurate $\mathrm{T}$ and $\mathrm{N}$ stage can be determined $[9,10]$. Dilation may be performed with either a Savary dilator over the wire, or with a through-the-scope (TTS) controlled radial expansion (CRE) dilation balloon. A higher rate of esophageal perforation has been reported with dilation of malignant esophageal strictures, but it is generally regarded as safe [11, 12]. In our practice, if we experience resistance due to a malignant stricture precluding passage of a standard EGD scope or echoendoscope, we dilate with a TTS CRE balloon up to 14-16 $\mathrm{mm}$ so as to then allow for a complete and thorough staging EUS exam. If a high-grade stricture is present, serial dilations may be necessary prior to the EUS staging.

\subsection{Determining the T stage}

Assessment of the depth of invasion of the tumor is necessary for determination of a $T$ stage. CT and MRI lack the sensitivity to accurately distinguish between the different layers of the esophageal wall. EUS has excellent sensitivity and specificity in assessing depth of invasion that increases with more advanced tumors (T4 tumors) [13]. The $\mathrm{T}$ stage for primary adenocarcinomas of the esophagus, esophagogastric junction (EGJ), and esophageal squamous cell cancers (SCC) are the same according to the $7^{\text {th }}$ Edition of the TNM staging manual of the American Joint Committee on Cancer (AJCC). EGJ tumors are those within the first $5 \mathrm{~cm}$ of the stomach that extend into the esophagogastric junction or distal thoracic esophagus. Non-anatomic classifications (histopathological cell type, histologic grade and tumor location) were identified in the latest revision of the manual for stage grouping [14]. Once the tumor has been identified endoscopically, the echoendoscope should be carefully advanced beyond the most distal portion of the tumor. Minimal balloon distention should be utilized to minimize compression of the tumor and esophageal wall. Esophageal tumors appear as hypoechoic masses with irregular borders and penetration beyond the esophageal layers should be described. Measurement of the mass thickness should be noted as it can predict extra-esophageal extension [15]. Early esophageal cancers are defined as tumors 
limited to the mucosa and/or submucosa but not extending into the muscular wall of the esophagus (T1)[16]. Tis is the earliest tumor stage and defined as high-grade dysplasia. It was previously known as carcinoma-in-situ and includes all noninvasive neoplastic epithelium [1, 14]. EUS is not accurate for mucosal evaluation and staging of these early lesions, even with the use of high-frequency endoscopic ultrasound probes. Tis lesions are best diagnosed by other means, such as endoscopic mucosal resection (EMR) [17].

Once a tumor has invaded the lamina propria, muscularis mucosa or submucosa but not the muscularis propria, it considered a stage T1 tumor. This stage is further sub-divided to T1a (also known as T1m and further subdivided to $\mathrm{m} 1$ : limited to the epithelial layer, $\mathrm{m} 2$ : invading lamina propria and $\mathrm{m} 3$ : invading into but not through muscularis mucosa) for tumors that invade the lamina propria or muscularis mucosa; and T1b (also known as T1sm and further subdivided into thirds to sm1, sm2 and sm3: deepest one third of the submucosa) for tumors that invade the submucosa $[1,13,18]$. The depth of invasion predicts the probability of lymph node metastasis, vascular invasion and long-term survival [19]. There has been much debate and controversy regarding the staging modality for early esophageal cancers. Although EUS can distinguish between stages T1 and $>\mathrm{T} 1$, recent data has shown that EUS is not sufficiently accurate in distinguishing between T1a and T1b tumors and pathological staging by EMR should be performed [17, 20,21]. Whether EUS should be performed prior to EMR is another debatable topic and beyond the scope of this chapter. Small caliber high-frequency EUS probes are able to visualize nine esophageal layers and may be able to accurately identify depth of invasion to guide therapy $[18,22,23]$.

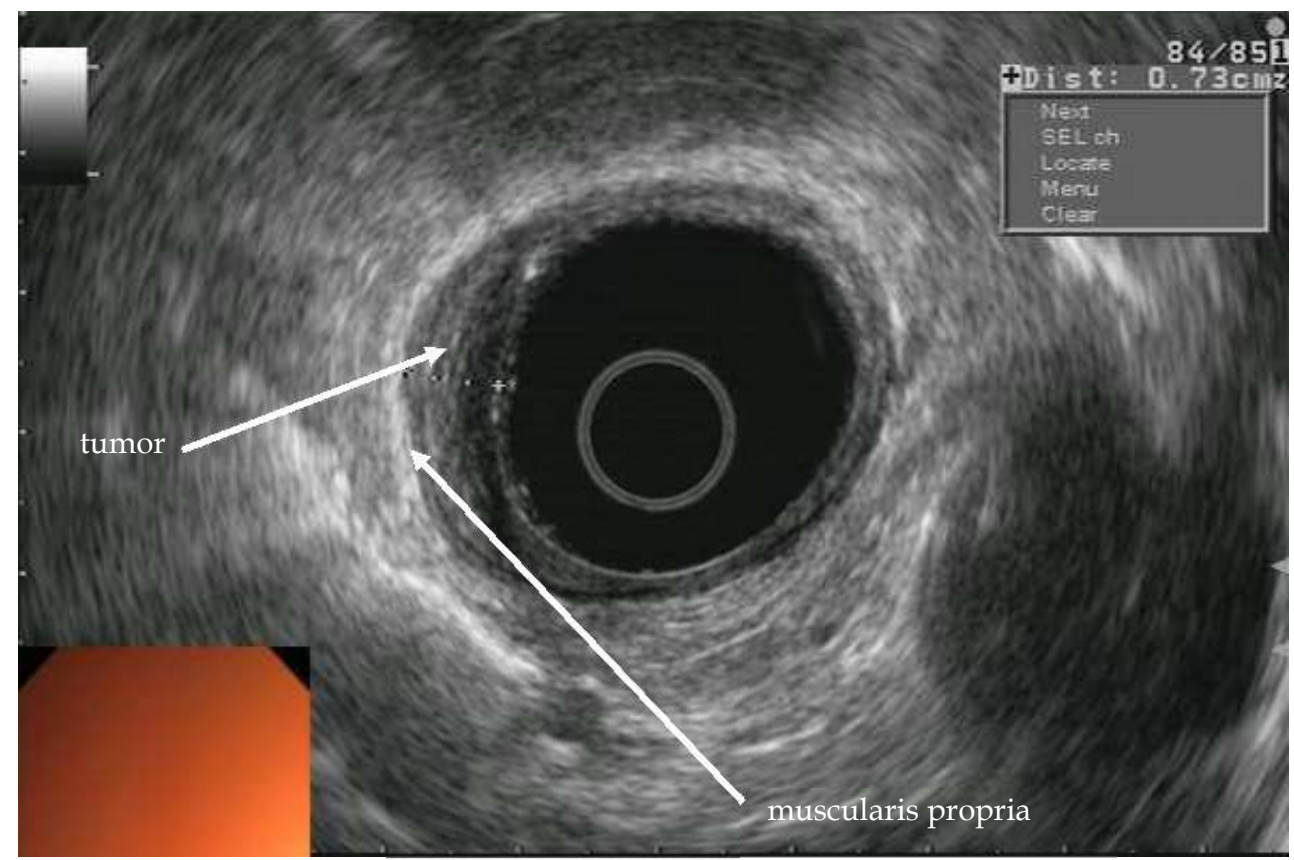

Image 2. T1 esophageal cancer; the muscularis propria can be clearly seen surrounding the tumor. 
Stage T2 tumors have invaded the fourth hypoechoic layer, the muscularis propria. In a recent meta-analysis, the diagnostic accuracy of EUS in staging of a T2 tumor was found to have a sensitivity of $81.4 \%$ and specificity of $96.3 \%$ [13]. The distinction between a T2 and T3 tumor is important in the decision for neoadjuvant therapy [24]. The endosonographer needs to be cautioned that overstaging of $\mathrm{T} 2$ tumors can lead to the inappropriate use of neoadjuvant therapy instead of immediate surgery [25].

EUS has its highest accuracy in staging advanced tumors. The distinction between a T3 (tumor invading adventitia, or going through the muscularis propria) and a T4 (tumor invading adjacent structures) is important in that it can determine resectability $[1,7,14]$. In the latest revision of the AJCC Staging Manual, T4 tumors were divided into resectable tumors (T4a) and unresectable (T4b). Those tumors invading pleura, pericardium and diaphragm are considered resectable and those invading other structures such as the trachea and aorta are considered unresectable $[1,26]$.

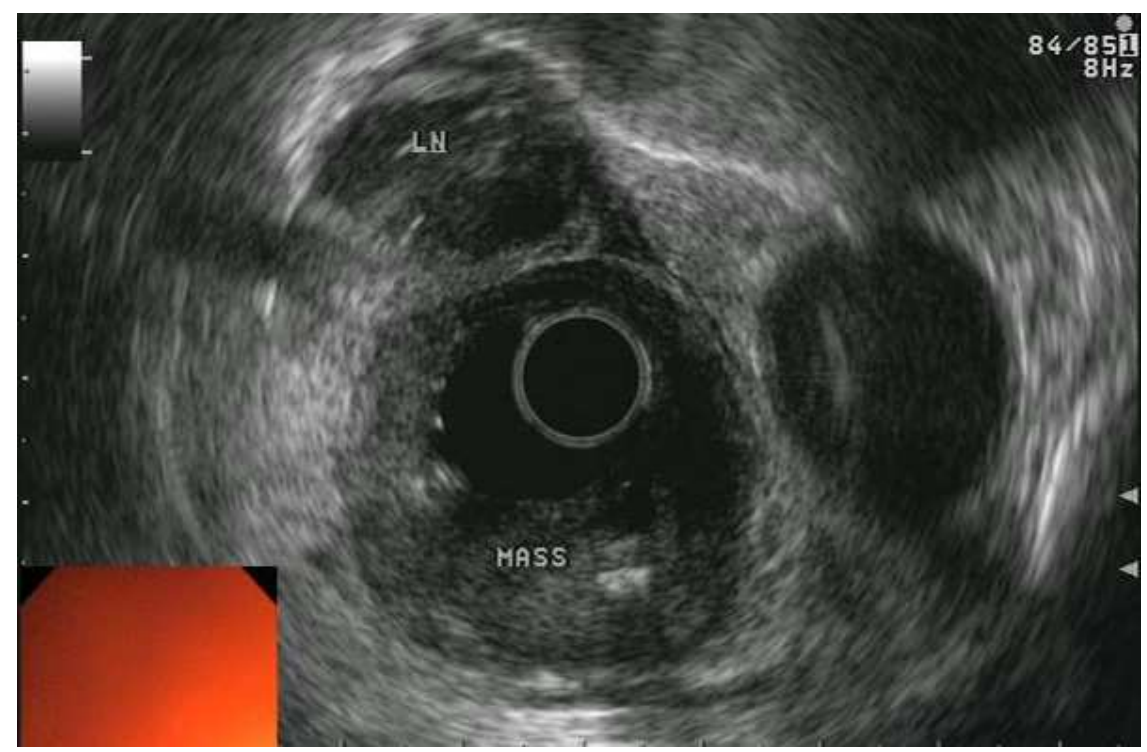

Image 3. T2 N1 Esophageal tumor; the mass can be seen invading through 4 of 5 esophageal layers. The adventitia is intact and there is no infiltration of tumor into adjacent structures.

A recent meta-analysis and systematic review of 49 studies showed EUS to have a high pooled sensitivity for $\mathrm{T}$ staging between $81-90 \%$ with a pooled specificity of approximately $99 \%$. The pooled sensitivity and specificity of EUS for assessing tumor depth per T stage were $81.6 \%$ and $99.4 \%$ for $\mathrm{T} 1,81.4 \%$ and $96.3 \%$ for $\mathrm{T} 2,91.4 \%$ and $94.4 \%$ for $\mathrm{T} 3$, and $92.4 \%$ and $97.4 \%$ for $\mathrm{T} 4$ [13].

\subsection{Determining the $\mathbf{N}$ stage}

EUS has a high accuracy for detecting regional lymph node involvement with a high sensitivity and specificity (85\% and $85 \%$ respectively in a recent meta-analysis). FNA significantly improves the diagnostic capability of EUS for detecting malignant lymph nodes (sensitivity of $97 \%$ and specificity of $96 \%$ ) by adding cytological analysis [13]. The 
characteristics of a malignant node are size greater than $1 \mathrm{~cm}$, rounded shape, sharp borders, and homogenous hypoechogenicity [5]. Malignant lymph nodes are usually in close proximity to the esophageal mass and often FNA can be impeded by the mass if in the trajectory of the needle. A selective approach to EUS-FNA may be applied by use of the modified EUS criteria for lymph node staging (standard characteristics plus EUS identified celiac lymph nodes, $>5$ lymph nodes, or EUS T3/4 tumor) for increased accuracy and decreased need for FNA (and cost) [27].

Regional lymph nodes are defined as any para-esophageal node extending from cervical nodes to celiac nodes. The number of involved lymph nodes--instead of their location--has been found to have prognostic implications [28-30]. This led to a revision of the $\mathrm{N}$ classification to support groupings of number of positive nodes as follows: N0 (none), N1 $(1-2)$, N2 (3-6), and N3 ( $\geq 7)$ [14]. The examination for metastatic lymph nodes begins with examination of the celiac axis and the diaphragmatic crurae and continues as the scope is withdrawn through the esophagus. Peri-tumoral lymph nodes should be noted during determination of the $\mathrm{T}$ stage and the remainder of the mediastinum should be carefully examined as the scope is withdrawn.

\subsection{Determining the $M$ stage}

The liver is the most common site of distant metastases of esophageal cancer. EUS can accurately evaluate the medial two thirds of the liver for metastases but cannot reliably exclude metastases in the entire liver [7]. CT and PET are most commonly used to evaluate for distant metastases. The role of EUS is greatest in confirming the presence of metastases in distant lymph nodes or lesions in the liver. The accuracy is increased by FNA and cytological evaluation of liver lesions as small as $4 \mathrm{~mm}$ [31-33]. Careful examination of the liver should be performed during staging for occult metastases not identified by other imaging studies. Identification of occult lesions may be low but can change the management of the patient. Any focal, discrete hypoechoic lesion of the liver identified should be sampled by EUS [34].

Malignant celiac lymph nodes are no longer considered metastatic and are grouped with regional lymph nodes. M classification is simply designated as M0 for no distant metastases, and M1 for presence of distant metastases [14].

\section{Restaging following neoadjuvant therapy}

The overall accuracy of EUS to assess the response to neoadjuvant chemotherapy or chemoradiotherapy is much less accurate than for initial staging and its role for this purpose is debatable [35, 36]. The presence of inflammation and fibrosis that remains following neoadjuvant therapy can be indistinguishable from residual tumor and may result in overstaging by EUS. Alternatively, residual microfoci of tumor in the esophageal wall may result in understaging [37]. Reduction of tumor thickness by $>50 \%$ is associated with a response to therapy and nodal status following neoadjuvant therapy has been shown to predict survival $[36,38,39]$.

\section{Staging of gastric tumors}

Patients with gastric cancer often present with advanced disease at the time of diagnosis, which is usually unresectable. Distant metastases and/or involvement of major blood vessels usually indicate unresectability. EUS is one of the primary means for loco-regional 
staging of gastric cancers. Patients typically present with abdominal pain, nausea, early satiety, anorexia and weight loss that warrants an initial upper endoscopic evaluation. In addition, gastric cancers are often diagnosed endoscopically when a CT scan is performed and notes a thickened gastric fold or a non-healing ulcer. EUS is then performed for further loco-regional staging.

The choice of echoendoscope for evaluation of gastric tumors is dependent on the location of the tumor and often a personal preference of the endosonographer. Obtaining adequate acoustic coupling between the gastric wall and the transducer can present a challenge. It is critical to remove all of the air from the stomach and instill up to $500 \mathrm{~mL}$ of water in order to adequately evaluate lesions (especially if the lesion is small). Changing the patient's position, such as placing the patient in a reverse Trendelenberg position to evaluate a lesion in the antrum, is often necessary to allow the water to pool in the targeted area. Care must be taken in preventing aspiration as these patients are sedated and thus possess a diminished gag reflex. A radial echoendoscope operating between 7.5 and $10 \mathrm{MHz}$ allows for adequate sonographic views of the gut wall with visualization of all 5 wall layers. Some endosonographers prefer a linear echoendoscope since it allows for FNA of lymph nodes without having to change endoscopes. Lastly, if a smaller lesion such as an early gastric cancer is encountered, a high-frequency ultrasound probe may provide the highest resolution to determine depth of invasion.

\subsection{Determining the T Stage}

EUS remains as the diagnostic tool of choice for evaluation of tumor depth in gastric cancer. This holds true especially in differentiating between early to intermediate (T1-2) and advanced (T3-4) primary tumors [40]. Gastric masses usually appear as irregular, poorlycircumscribed hypoechoic masses. The depth of invasion is determined by the penetration into one of the sonographic layers. It is important to note the integrity of each of these layers as described below.

The $7^{\text {th }}$ edition of the AJCC gastric cancer staging system applies to tumors arising in the more distal stomach, and those arising in the proximal $5 \mathrm{~cm}$ without crossing the esophagogastric junction [41]. Tumors confined to the mucosa and submucosa (T1), regardless of the $\mathrm{N}$ stage, are considered early gastric cancer [42]. Tis is the earliest stage and reserved for intraepithelial tumors without invasion of the lamina propria [1]. The T1 category is further subdivided into T1a for invasion of lamina propria or muscularis mucosa, and T1b for invasion of submucosa [41]. The distinction between Tis, T1a and T1b is important in deciding whether endoscopic resection is feasible. Contrary to staging in other parts of the GI tract, invasion of the lamina propria is classified as T1a rather than Tis since there is an abundance of lymphatic channels in the gastric mucosa, and thus associated lymph node metastases are possible when the tumor is confined to the lamina propria [41]. In areas with a high prevalence of gastric cancers, such as Japan and Korea, endoscopic mucosal resection (EMR) and endoscopic submucosal dissection (ESD) is widely employed as a safe and minimally invasive curative technique [43-46].

Invasion of the muscularis propria by tumor is considered T2 [1]. Sonographically, these lesions will involve the fourth layer (muscularis propria) without penetrating the fifth layer (serosa). Distinguishing between a T2 and T3 tumor can be difficult at times due to the subtle differences in the visualized gastric layers. Once the tumor has penetrated the fifth sonographic layer without invasion of visceral peritoneum or adjacent structures, it is classified as T3. In contrast to a T2 tumor, a T3 tumor will have irregular margins, often 
appearing as finger-like projections, or pseudopodia, extending into the gastrocolic or gastrohepatic ligaments, or into the greater or lesser omentum, without perforation of the visceral peritoneum covering these structures. T4 tumors are defined as those invading the serosa or adjacent structures, and they are divided into T4a for tumors invading the serosa (visceral peritoneum) and T4b for tumors invading adjacent structures such as spleen, transverse colon, liver, diaphragm, pancreas, abdominal wall, adrenal gland, kidney, small intestine, and retroperitoneum $[1,41]$.

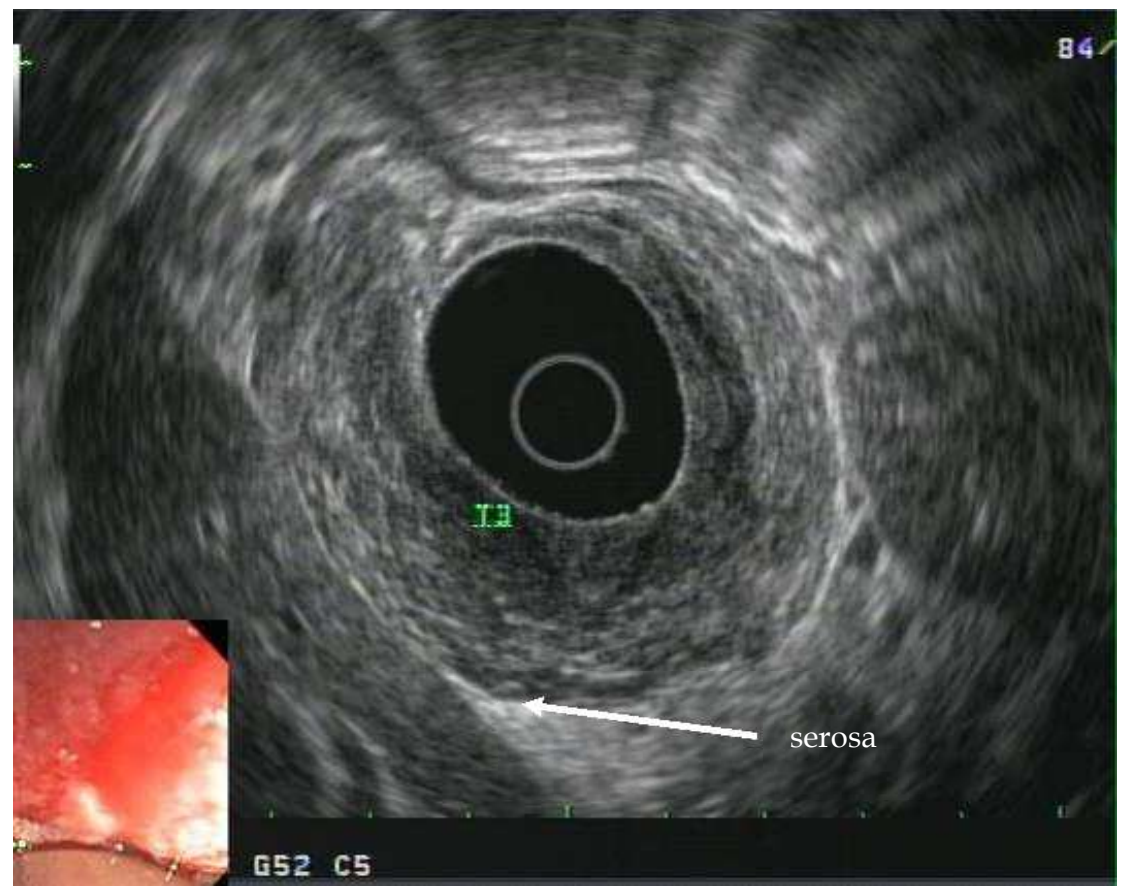

Image 4. T3 gastric adenocarcinoma with infiltration of the tumor beyond the $4^{\text {th }}$ sonographic layer without penetration of the visceral peritoneum.

A recent meta-analysis reviewed the available literature for the performance of EUS in staging of gastric cancer. The overall sensitivity for individual T stages ranged from $86 \%$ (for T3) to $65 \%$ for T2), and specificity of $96 \%$ (for T1) to $85 \%$ (for T3). An important observation of this meta-analysis was the high performance rates for differentiating between early to intermediate (T1-2) and advanced (T3-4) primary gastric tumors which may guide therapeutic management [40].

\subsection{Determining the $\mathrm{N}$ stage}

Similar to the newly adopted definition for $\mathrm{N}$ stage in staging of esophageal cancer, gastric cancer $\mathrm{N}$ stage classification is based on the number of regional lymph nodes as follows: N0 (none), N1 (1-2), N2 (3-6), N3 (7 or greater). The necessity to count the number of lymph nodes, as well as the heterogeneity in the criterion for nodes regarded as malignant, present a difficult challenge in assessing for nodal involvement. Scanning for peri-gastric lymph 
nodes should begin from the antrum to the gastroesophageal junction with the balloon distended and the gastric lumen compressed. Water can be instilled to aid in the visualization if a large amount of air artifact is seen. Malignant lymph nodes are usually regarded as round, hypoechoic, sharp and greater than $1 \mathrm{~cm}$ [5]. Although one study of resected gastrectomy specimens showed that $55 \%$ of metastasis-containing nodes were less than $5 \mathrm{~mm}$, suggesting that lymph node size is not a reliable predictor of metastases in gastric cancer [47].

\subsection{Determining the $M$ stage}

EUS is not suitable for detecting distant metastases but is sensitive in evaluating portions of the liver for metastatic disease and for malignant ascites. Various studies have shown that the detection of ascites by EUS in patients with gastric cancer is associated with peritoneal metastases [48-50]. Ascites appears as anechoic, triangular shaped collections of fluid in the peri-hepatic or peri-gastric regions. FNA can be formed for cytological evaluation. Care must be taken not to cross the tumor in order to obtain the fluid. This may produce a falsely positive result and also contaminate the fluid with malignant cells. Prophylactic antibiotics should be administered and continued post-procedure. Positive peritoneal cytology is classified as M1 [41].

\section{Infiltrating gastric malignancies}

Infiltrating malignancies of the stomach include the diffuse type of gastric adenocarcinoma (linitis plastica) and primary gastric lymphomas (PGL). EUS is important in determining the depth of involvement of these lesions. The normal gastric thickness is between 3 to $5 \mathrm{~mm}$ and appears thickened in infiltrating tumors. Sonographic images in linitis plastica will either show a homogenous appearance across all the layers of the gastric wall making the individual layers indistinguishable, or will appear as thickening of the third and fourth sonographic layer (submucosa and muscularis propria).

Benign conditions can often mimic malignant conditions by presenting with thickened gastric folds, such as in protein-losing hypertrophic gastropathy (Ménétrier's disease), amyloidosis and Zollinger-Ellison syndrome. EUS will usually reveal thickening limited to the first and second sonographic layer indicating a mucosal disease. Large capacity and jumbo forceps biopsy can often provide sufficient tissue to aid in the diagnosis of these disorders. For lesions involving the third and fourth sonographic layers, deep endoscopic biopsies (using a bite-on-bite technique) or full-thickness surgical biopsies are often necessary to make a diagnosis. A few case reports and studies have shown EUS-FNA as a feasible technique for obtaining tissue samples from thickened folds or other lesions within the GI tract wall, especially when endoscopic biopsies are negative. One retrospective study of EUS-FNA in evaluating intramural and extramural GI tract lesions showed the sensitivity, specificity, and diagnostic accuracy of EUS-FNA in diagnosing GI tract neoplastic lesions were $89 \%, 88 \%$, and $89 \%$, respectively[51].

The staging of PGL is different than for gastric adenocarcinoma and utilizes either the modified Ann Arbor staging system or the modified TNM staging system named the Paris classification [52, 53]. Sonographic appearance of PGL varies and can mimic other infiltrative diseases both benign and malignant. The appearance may be of a focal nodular infiltration of the mucosa, or as diffuse hypoechoic thickening of the mucosal layers (first 
and second sonographic layers) with fusion of these layers as the tumor extends [7, 54]. There is limited data in the literature regarding EUS for staging gastric lymphoma but it is generally accepted as the most accurate method to determine local stage. T stage accuracy has been reported between $80-92 \%$ and $\mathrm{N}$ stage between $77-90 \%$ [55]. FNA with flowcytometry of the aspirate may aid in the detection of metastatic lymph nodes and guide further management. The role of EUS in the follow-up of gastric lymphomas is not welldefined in the literature and we do not routinely employ its use in the absence of further studies.

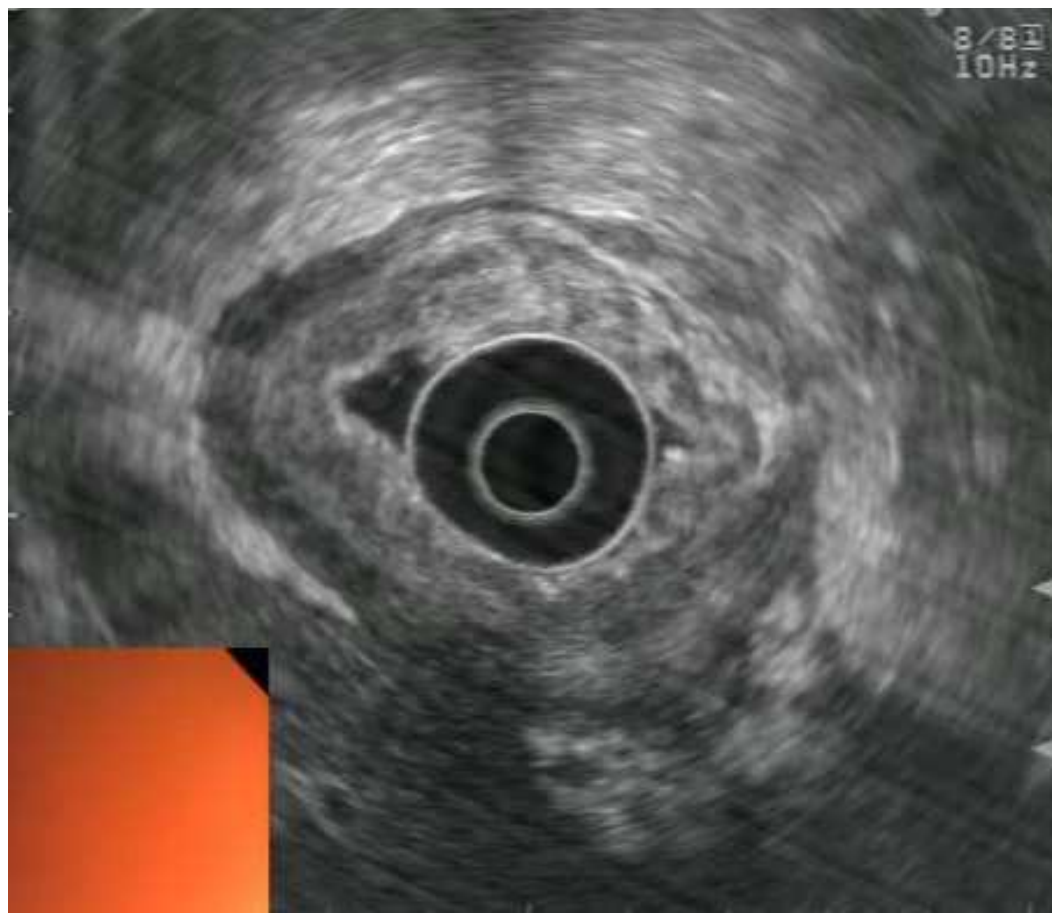

Image 5. Infiltrating gastric adenocarcinoma with thickening of the submucosa and muscularis propria.

\section{Staging of ampullary tumors}

Carcinomas of the ampulla of Vater are rare and can arise from the major papilla, pancreas, duodenum and the common bile duct. EUS is useful in evaluating the depth of invasion of ampullary tumors and it aids in determining whether endoscopic resection is feasible. Like colon polyps, these lesions follow the adenoma-carcinoma sequence. Benign adenomas of the ampulla should be removed entirely by endoscopic ampullectomy. Conversely, malignant or invasive lesions should be removed surgically, often requiring a pancreaticoduodenectomy for complete resection. Patients with ampullary carcinomas typically present with obstructive jaundice or pancreatitis. Occasionally they are found incidentally by upper endoscopy. 
Classification according to the 7th Edition of the AJCC staging system for tumors of the ampulla of Vater is as follows: Tis corresponds to carcinoma in situ, T1 tumors are limited to the ampulla of Vater or sphincter of Oddi, T2 tumors invade the duodenal wall, T3 tumors invade the pancreas, and T4 tumors invade peri-pancreatic soft tissues or other adjacent organs or structures other than the pancreas[1].

Sonographic imaging of the ampulla of Vater can be performed either with a radial or linear-array echoendoscope. Once the echoendoscope is advanced to the second portion of the duodenum, it should be withdrawn slowly while scanning for the ampullary lesion. The balloon should be filled just enough as not to press on the ampullary lesion and water should be instilled into the duodenum for improved echogenic coupling. An alternative method is to visualize the ampullary lesion endoscopically and place the water-filled balloon directly on the lesion. A combination of both approaches may yield the best results. The ampulla appears as a hypoechoic structure arising from the wall of the duodenum usually measuring 8 to $12 \mathrm{~mm}$. Ampullary tumors are hypoechoic masses at the ampulla which create loss of interface between the different echogenic layers of the duodenal wall. The sphincter of Oddi may be difficult to visualize but would appear as a thin hypoechoic layer surrounding the pancreaticobiliary duct. Extension of the hypoechoic mass within the biliary or pancreatic duct lumen, or wall thickening of the duct, suggests ductal infiltration $[7,56]$.

The diagnostic accuracy of EUS in ampullary tumor staging is reportedly $0-100 \%$ for T1, 45$100 \%$ for T2, and $75-100 \%$ for T3-T4 lesions with an overall accuracy in tumor staging from 62 and 90\% [57]. Decreased accuracy and understaging has been reported when a biliary stent is present.

Intraductal ultrasound (IDUS) is yet another method of visualizing ampullary tumors by use of a high-frequency ultrasound probe (20-30 MHz) inserted into the bile duct during ERCP. Various studies have shown superior T staging for IDUS compared to EUS [56, 58, 59], with overall accuracy for tumor staging ranging between $78-88 \%$ for IDUS. Limitations of IDUS include the necessity of ERCP for staging and cannulation of the bile duct (which may be difficult with larger tumors), cost of the probe, potential damage to the probe by the duodenoscope elevator, and its limited availability.

Nodal metastases are best evaluated by EUS as it has been shown to have superior accuracy for detection of malignant lymph nodes compared to transabdominal ultrasound and CT [60-62]. MRI may show equal or improved lymph node detection. CT is superior for detection of distant metastases. The technique for malignant lymph node detection involves scanning the peri-pancreatic regions for any suspicious nodes. Regional lymph nodes (N1) are peri-pancreatic nodes including: hepatic, hepatic artery, epiploic, omental, peri-portal, infra-pyloric, celiac, superior mesenteric, retroperitoneal, and lateral aortic (lumbar) nodes. Tumor involvement of other nodal groups such as splenic and para-aortic lymph nodes and those at the tail of the pancreas are not regional and classified as distant metastases (M1) [1]. FNA can be performed of any malignant appearing lymph nodes for cytological analysis.

\section{Staging of duodenal tumors}

Non-ampullary duodenal adenocarcinomas are exceedingly rare accounting for $1-2 \%$ of all GI malignancies and $25-50 \%$ of all small intestinal cancers. Adenocarcinomas are the most common followed by carcinoid tumors $[1,63,64]$. 
Duodenal tumors are staged using the 7th edition of the AJCC for Small Intestine Cancer.[1] The role of EUS in staging these tumors is not well-defined and has not been widely studied. Depending on the size of the tumor, a radial echoendoscope or a high-frequency ultrasound probe can be utilized to assess depth of invasion for $\mathrm{T}$ staging. In order to obtain accurate imaging of the 5 sonographic layers of the duodenal wall, all of the air should be aspirated out of the lumen and the tumor should be submerged in water.

Nodal status is an important predictive factor for recurrence and survival in patients with resectable duodenal adenocarcinoma [64]. EUS can aid in the assessment of regional lymph nodes by counting and possibly performing FNA of suspicious nodes.

\section{References}

[1] Edge, S.B. and American Joint Committee on Cancer., AJCC cancer staging manual. 7th ed2010, New York ; London: Springer. xiv, 648 p.

[2] Liu, J., et al., Endoscopic ultrasound probes. Gastrointestinal endoscopy, 2006. 63(6): p. 751-4.

[3] Kimmey, M.B., et al., Histologic correlates of gastrointestinal ultrasound images. Gastroenterology, 1989. 96(2): p. 433-441.

[4] Aibe, T., et al., A Fundamental Study of Normal Layer Structure of the Gastrointestinal Wall Visualized by Endoscopic Ultrasonography. Scandinavian journal of gastroenterology, 1986. 21(s123): p. 6-15.

[5] Catalano, M.F., et al., Endosonographic features predictive of lymph node metastasis. Gastrointestinal endoscopy, 1994. 40(4): p. 442-6.

[6] Chen, V.K. and M.A. Eloubeidi, Endoscopic ultrasound-guided fine needle aspiration is superior to lymph node echofeatures: a prospective evaluation of mediastinal and periintestinal lymphadenopathy. The American journal of gastroenterology, 2004. 99(4): p. 628-33.

[7] Gress, F.G. and T.J. Savides, Endoscopic ultrasonography. 2nd ed2009, Chichester, West Sussex, UK ; Hoboken, NJ: Wiley-Blackwell. xiii, 202 p.

[8] Low, D., Update on Staging and Surgical Treatment Options for Esophageal Cancer. Journal of Gastrointestinal Surgery, 2011: p. 1-11.

[9] Morgan, M.A., et al., Prognostic significance of failure to cross esophageal tumors by endoluminal ultrasound. Diseases of the Esophagus, 2008. 21(6): p. 508-513.

[10] Jacobson, B.C., et al., The role of endoscopy in the assessment and treatment of esophageal cancer. Gastrointestinal endoscopy, 2003. 57(7): p. 817-822.

[11] Kallimanis, G.E., et al., Endoscopic ultrasound for staging esophageal cancer, with or without dilation, is clinically important and safe. Gastrointestinal endoscopy, 1995. 41(6): p. 540-546.

[12] Jacobson, B., et al., Through-the-Scope Balloon Dilation for Endoscopic Ultrasound Staging of Stenosing Esophageal Cancer. Digestive diseases and sciences, 2007. 52(3): p. 817822.

[13] Puli, S.R., et al., Staging accuracy of esophageal cancer by endoscopic ultrasound: a metaanalysis and systematic review. World journal of gastroenterology : WJG, 2008. 14(10): p. $1479-90$. 
[14] Rice, T., E. Blackstone, and V. Rusch, 7th Edition of the AJCC Elt;i\&gt;Cancer Staging Manual:Elt;/iEgt; Esophagus and Esophagogastric Junction. Annals of surgical oncology, 2010. 17(7): p. 1721-1724.

[15] Brugge, W.R., et al., Endoscopic ultrasound staging criteria for esophageal cancer. Gastrointestinal endoscopy, 1997. 45(2): p. 147-152.

[16] Stein, H.J., et al., Early esophageal cancer: pattern of lymphatic spread and prognostic factors for long-term survival after surgical resection. Annals of surgery, 2005. 242(4): p. 56673; discussion 573-5.

[17] Young, P.E., et al., Endoscopic Ultrasound Does Not Accurately Stage Early Adenocarcinoma or High-Grade Dysplasia of the Esophagus. Clinical gastroenterology and hepatology : the official clinical practice journal of the American Gastroenterological Association, 2010. 8(12): p. 1037-1041.

[18] Attila, T. and D.O. Faigel, Role of endoscopic ultrasound in superficial esophageal cancer. Diseases of the Esophagus, 2009. 22(2): p. 104-112.

[19] Endo, et al., Clinicopathologic analysis of lymph node metastasis in surgically resected superficial cancer of the thoracic esophagus. Diseases of the Esophagus, 2000. 13(2): p. 125-129.

[20] Pouw, R.E., et al., Do we still need EUS in the workup of patients with early esophageal neoplasia? A retrospective analysis of 131 cases. Gastrointestinal endoscopy, 2011. 73(4): p. 662-668.

[21] Pech, O., et al., The Impact of Endoscopic Ultrasound and Computed Tomography on the TNM Staging of Early Cancer in Barrett's Esophagus. The American journal of gastroenterology, 2006. 101(10): p. 2223-2229.

[22] Hasegawa, N., et al., Preoperative staging of superficial esophageal carcinoma: comparison of an ultrasound probe and standard endoscopic ultrasonography. Gastrointestinal endoscopy, 1996. 44(4): p. 388-393.

[23] Murata, Y., et al., Small ultrasonic probes for determination of the depth of superficial esophageal cancer. Gastrointestinal endoscopy, 1996. 44(1): p. 23-28.

[24] Gebski, V., et al., Survival benefits from neoadjuvant chemoradiotherapy or chemotherapy in oesophageal carcinoma: a meta-analysis. The lancet oncology, 2007. 8(3): p. 226-34.

[25] Pech, O., et al., Accuracy of endoscopic ultrasound in preoperative staging of esophageal cancer: results from a referral center for early esophageal cancer. Endoscopy, 2010. 42(6): p. 45661.

[26] Rice, T.W., et al., Cancer of the esophagus and esophagogastric junction. Cancer, 2010. 116(16): p. 3763-3773.

[27] Vazquez-Sequeiros, E., et al., Routine vs. selective EUS-guided FNA approach for preoperative nodal staging of esophageal carcinoma. Gastrointestinal endoscopy, 2006. 63(2): p. 204-211.

[28] Rizk, N., et al., The prognostic importance of the number of involved lymph nodes in esophageal cancer: Implications for revisions of the American Joint Committee on Cancer staging system. The Journal of Thoracic and Cardiovascular Surgery, 2006. 132(6): p. 13741381.e2.

[29] Mariette, C., et al., The number of metastatic lymph nodes and the ratio between metastatic and examined lymph nodes are independent prognostic factors in esophageal cancer regardless 
of neoadjuvant chemoradiation or lymphadenectomy extent. Annals of surgery, 2008. 247(2): p. 365-71.

[30] Zhang, H.L., et al., The number of lymph node metastases influences survival and International Union Against Cancer tumor-node-metastasis classification for esophageal squamous cell carcinoma. Diseases of the Esophagus, 2010. 23(1): p. 53-58.

[31] Puli, S.R., et al., Accuracy of endoscopic ultrasound in the diagnosis of distal and celiac axis lymph node metastasis in esophageal cancer: a meta-analysis and systematic review. Digestive diseases and sciences, 2008. 53(9): p. 2405-14.

[32] Singh, P., et al., Endoscopic ultrasound versus CT scan for detection of the metastases to the liver: results of a prospective comparative study. Journal of clinical gastroenterology, 2009. 43(4): p. 367-73.

[33] van Vliet, E.P.M., et al., Staging investigations for oesophageal cancer: a meta-analysis. Br J Cancer, 2008. 98(3): p. 547-557.

[34] Prasad, P., et al., Detection of occult liver metastases during EUS for staging of malignancies. Gastrointestinal endoscopy, 2004. 59(1): p. 49-53.

[35] Jamil, L.H., K.R. Gill, and M.B. Wallace, Staging and restaging of advanced esophageal cancer. Current opinion in gastroenterology, 2008. 24(4): p. 530-4.

[36] Ribeiro, A., et al., Endoscopic ultrasound restaging after neoadjuvant chemotherapy in esophageal cancer. The American journal of gastroenterology, 2006. 101(6): p. 1216-21.

[37] Lightdale, C.J. and K.G. Kulkarni, Role of endoscopic ultrasonography in the staging and follow-up of esophageal cancer. Journal of clinical oncology : official journal of the American Society of Clinical Oncology, 2005. 23(20): p. 4483-9.

[38] Mesenas, S., et al., A large series, resection controlled study to assess the value of radial EUS in restaging gastroesophageal cancer following neoadjuvant chemotherapy. Diseases of the esophagus : official journal of the International Society for Diseases of the Esophagus / I.S.D.E, 2008. 21(1): p. 37-42.

[39] Kalha, I., et al., The accuracy of endoscopic ultrasound for restaging esophageal carcinoma after chemoradiation therapy. Cancer, 2004. 101(5): p. 940-7.

[40] Mocellin, S., A. Marchet, and D. Nitti, EUS for the staging of gastric cancer: a meta-analysis. Gastrointestinal endoscopy. In Press, Corrected Proof.

[41] Washington, K., 7th edition of the AJCC cancer staging manual: stomach. Annals of surgical oncology, 2010. 17(12): p. 3077-9.

[42] Tsuzuki, T., et al., Usefulness and problems of endoscopic ultrasonography in prediction of the depth of tumor invasion in early gastric cancer. Acta medica Okayama, 2011. 65(2): p. 105-12.

[43] Choi, J., et al., Endoscopic prediction of tumor invasion depth in early gastric cancer. Gastrointestinal endoscopy, 2011. 73(5): p. 917-927.

[44] Isomoto, H., et al., Endoscopic submucosal dissection for early gastric cancer: a large-scale feasibility study. Gut, 2009. 58(3): p. 331-336.

[45] Chung, I.I.K., et al., Therapeutic outcomes in 1000 cases of endoscopic submucosal dissection for early gastric neoplasms: Korean ESD Study Group multicenter study. Gastrointestinal endoscopy, 2009. 69(7): p. 1228-1235. 
[46] Choi, J., et al., Comparison of endoscopic ultrasonography and conventional endoscopy for prediction of depth of tumor invasion in early gastric cancer. Endoscopy, 2010. 42(9): p. 705-13.

[47] Monig, S., et al., Staging of gastric cancer: correlation of lymph node size and metastatic infiltration. Am. J. Roentgenol., 1999. 173(2): p. 365-367.

[48] Lee, Y.T., et al., Accuracy of endoscopic ultrasonography in diagnosing ascites and predicting peritoneal metastases in gastric cancer patients. Gut, 2005. 54(11): p. 1541-1545.

[49] Chu, K.-M., et al., A prospective evaluation of catheter probe EUS for the detection of ascites in patients with gastric carcinoma. Gastrointestinal endoscopy, 2004. 59(4): p. 471474.

[50] Kaushik, N., et al., EUS-guided paracentesis for the diagnosis of malignant ascites. Gastrointestinal endoscopy, 2006. 64(6): p. 908-913.

[51] Vander Noot, M.R., 3rd, et al., Diagnosis of gastrointestinal tract lesions by endoscopic ultrasound-guided fine-needle aspiration biopsy. Cancer, 2004. 102(3): p. 157-63.

[52] Radaszkiewicz, T., B. Dragosics, and P. Bauer, Gastrointestinal malignant lymphomas of the mucosa-associated lymphoid tissue: factors relevant to prognosis. Gastroenterology, 1992. 102(5): p. 1628-38.

[53] Ruskoné-Fourmestraux, A., et al., Paris staging system for primary gastrointestinal lymphomas. Gut, 2003. 52(6): p. 912-913.

[54] Fischbach, W. and O. Al-Taie, Staging role of EUS. Best Practice \& Research Clinical Gastroenterology, 2010. 24(1): p. 13-17.

[55] Janssen, J., The impact of EUS in primary gastric lymphoma. Best Practice \& Research Clinical Gastroenterology, 2009. 23(5): p. 671-678.

[56] Ito, K., et al., Preoperative evaluation of ampullary neoplasm with EUS and transpapillary intraductal US: a prospective and histopathologically controlled study. Gastrointestinal endoscopy, 2007. 66(4): p. 740-7.

[57] Ito, K., et al., Diagnosis of Ampullary Cancer. Digestive surgery, 2010. 27(2): p. 115-118.

[58] Itoh, A., et al., Intraductal ultrasonography in diagnosing tumor extension of cancer of the papilla of Vater. Gastrointestinal endoscopy, 1997. 45(3): p. 251-60.

[59] Menzel, J., et al., Polypoid tumors of the major duodenal papilla: preoperative staging with intraductal US, EUS, and CT--a prospective, histopathologically controlled study. Gastrointestinal endoscopy, 1999. 49(3 Pt 1): p. 349-57.

[60] Chen, C.H., et al., Reappraisal of endosonography of ampullary tumors: correlation with transabdominal sonography, CT, and MRI. Journal of clinical ultrasound : JCU, 2009. 37(1): p. 18-25.

[61] Chen, C.-H., et al., Preoperative evaluation of periampullary tumors by endoscopic sonography, transabdominal sonography, and computed tomography. Journal of Clinical Ultrasound, 2001. 29(6): p. 313-321.

[62] Chen, C.H., et al., The accuracy of endoscopic ultrasound, endoscopic retrograde cholangiopancreatography, computed tomography, and transabdominal ultrasound in the detection and staging of primary ampullary tumors. Hepato-gastroenterology, 2001. 48(42): p. 1750-3. 
[63] Chung, W.C., et al., Prognostic factors associated with survival in patients with primary duodenal adenocarcinoma. The Korean journal of internal medicine, 2011. 26(1): p. 34-40.

[64] Struck, A., et al., Non-ampullary duodenal adenocarcinoma: factors important for relapse and survival. Journal of surgical oncology, 2009. 100(2): p. 144-8. 


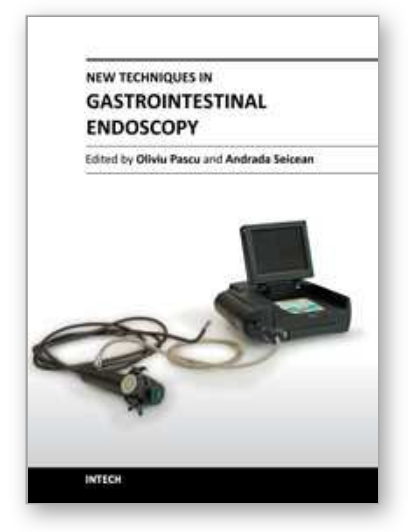

\author{
New Techniques in Gastrointestinal Endoscopy \\ Edited by Prof. Oliviu Pascu
}

ISBN 978-953-307-777-2

Hard cover, 310 pages

Publisher InTech

Published online 30, September, 2011

Published in print edition September, 2011

As result of progress, endoscopy has became more complex, using more sophisticated devices and has claimed a special form. In this moment, the gastroenterologist performing endoscopy has to be an expert in macroscopic view of the lesions in the gut, with good skills for using standard endoscopes, with good experience in ultrasound (for performing endoscopic ultrasound), with pathology experience for confocal examination. It is compulsory to get experience and to have patience and attention for the follow-up of thousands of images transmitted during capsule endoscopy or to have knowledge in physics necessary for autofluorescence imaging endoscopy. Therefore, the idea of an endoscopist has changed. Examinations mentioned need a special formation, a superior level of instruction, accessible to those who have already gained enough experience in basic diagnostic endoscopy. This is the reason for what these new issues of endoscopy are presented in this book of New techniques in Gastrointestinal Endoscopy.

\title{
How to reference
}

In order to correctly reference this scholarly work, feel free to copy and paste the following:

Juan Carlos Bucobo and Jonathan M. Buscaglia (2011). EUS Staging of Luminal Cancers in the Upper GI Tract, New Techniques in Gastrointestinal Endoscopy, Prof. Oliviu Pascu (Ed.), ISBN: 978-953-307-777-2, InTech, Available from: http://www.intechopen.com/books/new-techniques-in-gastrointestinal-endoscopy/eusstaging-of-luminal-cancers-in-the-upper-gi-tract

\section{INTECH}

open science | open minds

\author{
InTech Europe \\ University Campus STeP Ri \\ Slavka Krautzeka 83/A \\ 51000 Rijeka, Croatia \\ Phone: +385 (51) 770447 \\ Fax: +385 (51) 686166 \\ www.intechopen.com
}

\author{
InTech China \\ Unit 405, Office Block, Hotel Equatorial Shanghai \\ No.65, Yan An Road (West), Shanghai, 200040, China \\ 中国上海市延安西路65号上海国际贵都大饭店办公楼 405 单元 \\ Phone: +86-21-62489820 \\ Fax: +86-21-62489821
}


(C) 2011 The Author(s). Licensee IntechOpen. This chapter is distributed under the terms of the Creative Commons Attribution-NonCommercialShareAlike-3.0 License, which permits use, distribution and reproduction for non-commercial purposes, provided the original is properly cited and derivative works building on this content are distributed under the same license. 